\title{
Biosynthesis of the Envelope Glycolipids during Heterocyst Development in Anabaena cylindrica
}

\author{
By WITOLD J. KREPSKI† AND TERENCE J. WALTON* \\ Department of Biochemistry, University College of Swansea, Singleton Park, \\ Swansea SA2 8PP, U.K.
}

(Received 5 April 1982)

\begin{abstract}
Incorporation of sodium $\left[1^{-14} \mathrm{C}\right]$ acetate into the heterocyst-specific glycolipids and glycerolipids of the cyanobacterium Anabaena cylindrica during induced heterocyst differentiation indicated that (1) the activity of key enzymes involved specifically in the biosynthesis of the very long chain polyhydroxyalkanes and hydroxyfatty acid moieties of the heterocyst glycolipid fraction is stimulated during heterocyst differentiation, and (2) in mature heterocysts, formation of the characteristic very long chain heterocyst glycolipids essentially ceases.
\end{abstract}

\section{INTRODUCTION}

When grown in the absence of a combined nitrogen source, vegetative cells of Anabaena cylindrica undergo characteristic morphological changes, associated with changes in their physiology, to give rise to the characteristic pattern of heterocysts observed in the cyanobacterial filament (Haselkorn, 1978; Fay, 1980). Prominent amongst the morphological changes is the development of the refractile multi-layered heterocyst envelope, the innermost layer of which (the 'laminated' layer) is composed of a heterogeneous family of structurally related glycolipids. The semi-crystalline arrangement of these has been suggested to present a barrier to the ready diffusion of oxygen from the growth medium into the heterocyst, thus protecting the oxygen-sensitive nitrogenase system (Winkenbach et al., 1972). Deposition of the laminated layer appears to be a necessary requirement for development of heterocysts capable of aerobic dinitrogen fixation, since mutants of Anabaena variabilis deficient in heterocyst envelope glycolipids have no or very little nitrogenase activity when assayed aerobically (Haury \& Wolk, 1978).

The glycolipids produced are apparently unique to cyanobacteria capable of nitrogen fixation and are absent from cyanobacterial species incapable of nitrogen fixation and a diverse range of eukaryotic algae (Nichols \& Wood, 1968). In species such as $A$. cylindrica they are produced only when the organism is grown in a medium lacking an assimilable fixed nitrogen source, and are concentrated, if not located exclusively, in the heterocyst (Walsby \& Nichols, 1969). The major components of this heterocyst glycolipid (HG) fraction are glycosides of $\mathrm{C}_{26}$ and $\mathrm{C}_{28}$ polyhydroxyalkanes, and glycose esters of $\mathrm{C}_{26}$ and $\mathrm{C}_{28}$ hydroxyfatty acids (Bryce et al., 1972; Lambein \& Wolk, 1973). The similarity in structure of these very long chain hydroxyfatty acids and polyhydroxyalkanes suggests a precursor-product relationship. The structure of these lipids is, however, in marked contrast to those of the polar glycerolipids of the heterocysts and vegetative cells in which monogalactosyldiacylglycerol (MGDG), digalactosyldiacylglycerol (DGDG), phosphatidylglycerol (PG) and sulphoquinovosyldiacylglycerol (SQDG) are the major lipids, containing principally $\mathrm{C}_{16}$ and $\mathrm{C}_{18}$ fatty acyl substituents (Winkenbach et al., 1972; Zepke et al., 1978).

† Present address: FBC Limited, Chesterford Park Research Station, Saffron Walden, Essex CB10 1XL, U.K.

$\bar{A} \bar{b}$ breviations : $\overline{\mathrm{D}} \overline{\mathrm{D}} \overline{\mathrm{DG}} \overline{\mathrm{g}}$, digalactosyldiacylglycerol; $\mathrm{HG}$, heterocyst glycolipid; MGDG, monogalactosyldiacylglycerol; PG, phosphatidylglycerol; SQDG, sulphoquinovosyldiacylglycerol. 
Since very long chain $\left(>\mathrm{C}_{20}\right)$ fatty acids and their derivatives are absent from the vegetative cells of $A$. cylindrica, biosynthesis of the aglycone moieties of the HG fraction must be induced during heterocyst differentiation. Heterocyst differentiation, induced by transfer of cells grown in medium containing combined nitrogen to a medium lacking a combined nitrogen source (Bradley \& Carr, 1976) has been used to extend studies of the biosynthesis of the characteristic very long chain hydroxyfatty acids and polyhydroxyalkanes which constitute the aglycone moieties of the HG fraction and its relationship with glycerolipid fatty acid formation.

\section{METHODS}

Organism. Anabaena cylindrica 1430/2a was obtained from the Culture Centre of Algae and Protozoa, Cambridge, U.K.

Growth. Batch cultures were maintained in modified ASM-1 mineral salt medium, as described elsewhere (Abreu-Grobois et al., 1977). Under aseptic conditions, after $40 \mathrm{~h}$ growth in this medium, batch cultures were supplemented with $5 \mathrm{M}-\mathrm{NH}_{4} \mathrm{Cl}$ to give $5 \mathrm{mM}-\mathrm{NH}_{4}$ in the growth medium to suppress further heterocyst formation; these cultures are referred to as being grown in combined nitrogen medium. Batch cultures of $15 \mathrm{I}$ were grown in 201 vessels; for cultures grown in combined nitrogen medium, the vessel was modified to accept a pH electrode and the $\mathrm{pH}$ of the medium constantly monitored and maintained at $\mathrm{pH} 7.8$ throughout growth by addition of $200 \mathrm{mM}-\mathrm{KOH}$. Cultures were continuously aerated and agitated with compressed air [0.5 1 air ( 1 medium $\left.)^{-1} \mathrm{~min}^{-1}\right]$ and grown under constant illumination $(2500 \mathrm{~lx})$ at $25^{\circ} \mathrm{C}$.

After $226 \mathrm{~h}$ growth in combined nitrogen medium, cells $(2 \mathrm{l})$ were harvested by centrifugation at $4000 \mathrm{~g}$ for $10 \mathrm{~min}$ at $20^{\circ} \mathrm{C}$ and transferred aseptically to nitrogen-free medium (2l) and resuspended.

Total heterocyst frequency, chlorophyll content and nitrogen fixation assays. Total heterocyst frequency was determined by counting at $\times \mathbf{4 0 0}$ magnification under a light microscope. Chlorophyll content and nitrogen fixation assays were determined spectrophotometrically and by the GLC-acetylene reduction technique, respectively, as described previously (Krepski \& Walton, 1979). All assays were carried out on cells harvested by centrifugation (as described above) from $200 \mathrm{ml}$ growth medium.

Radioactive incubations. Cells from $200 \mathrm{ml}$ growth medium were harvested by centrifugation as described above and resuspended in $2 \mathrm{ml}$ of the appropriate growth medium containing $92.5 \mathrm{kBq}$ sodium [1-14 $\mathrm{C}$ acetate (specific activity $2.1 \mathrm{TBq} \mathrm{mol}^{-1}$ ) obtained from Amersham. The mixture was then incubated in a gyratory shaker (100 r.p.m.) at $25^{\circ} \mathrm{C}$ under constant illumination $(2500 \mathrm{~lx})$ for $3 \mathrm{~h}$. Incubations were terminated by addition of propan-2-ol (15 ml).

Extraction and analysis of lipids. Total lipid was extracted and examined by radio-TLC as described by AbreuGrobois et al. (1977). HG fractions were isolated by preparative TLC and subjected to alkaline hydrolysis (2 Mmethanolic $\mathrm{KOH}$ for $3 \mathrm{~h}$ at ambient temperature) and acid hydrolysis [refluxed with $2 \mathrm{M}^{-} \mathrm{H}_{2} \mathrm{SO}_{4}$ in methanol/water $(9: 1, \mathrm{v} / \mathrm{v})$ for $28 \mathrm{~h}$ ]. Fatty acid methyl esters from the HG ester fraction and the total lipid were prepared and analysed for radioactivity as described previously (Krepski \& Walton, 1979) and the aglycone fraction of the glycoside HG components was analysed by radio-TLC (Abreu-Grobois et al., 1977).

\section{RESULTS AND DISCUSSION}

Transfer of cells from a combined nitrogen medium to a nitrogen-free medium resulted in the expected increase in heterocyst frequency which was greatest for the first $30 \mathrm{~h}$ following transfer. During this period there was no significant increase in chlorophyll content of the culture (Fig. 1a), indicating that the observed increase in heterocyst frequency was due to induced differentiation of existing cells rather than new growth. The highest rate of nitrogenase activity was observed $55 \mathrm{~h}$ after transfer (Fig. $1 b$ ) and was coincident with the maximal heterocyst frequency (Fig. 1a). Increased labelling of the HG fraction was observed $6 \mathrm{~h}$ after transfer, at which time there was no significant increase in nitrogenase activity. Maximum synthesis of HG preceded the highest rates of nitrogen fixation by some $22 \mathrm{~h}$ and thereafter declined sharply, returning to levels comparable with that seen at transfer, while nitrogenase activity remained relatively high (Fig. $1 b$ ), supporting the proposal that presence of $\mathrm{HG}$ is an essential prerequisite for aerobic nitrogen fixation in physiologically mature heterocysts (Haury \& Wolk, 1978).

The stimulatory effect on HG biosynthesis observed $6 \mathrm{~h}$ after transfer to nitrogen-free medium was accompanied by smaller increases in the level of labelling of the MGDG, SQDG and DGDG, and PG fractions; however, while HG labelling continued to increase until $28 \mathrm{~h}$ after transfer, labelling of the glycerolipids fell after $6 \mathrm{~h}$ and gradually returned to the levels observed at the time of transfer (Fig. 2). The labelling pattern and time course clearly indicate 


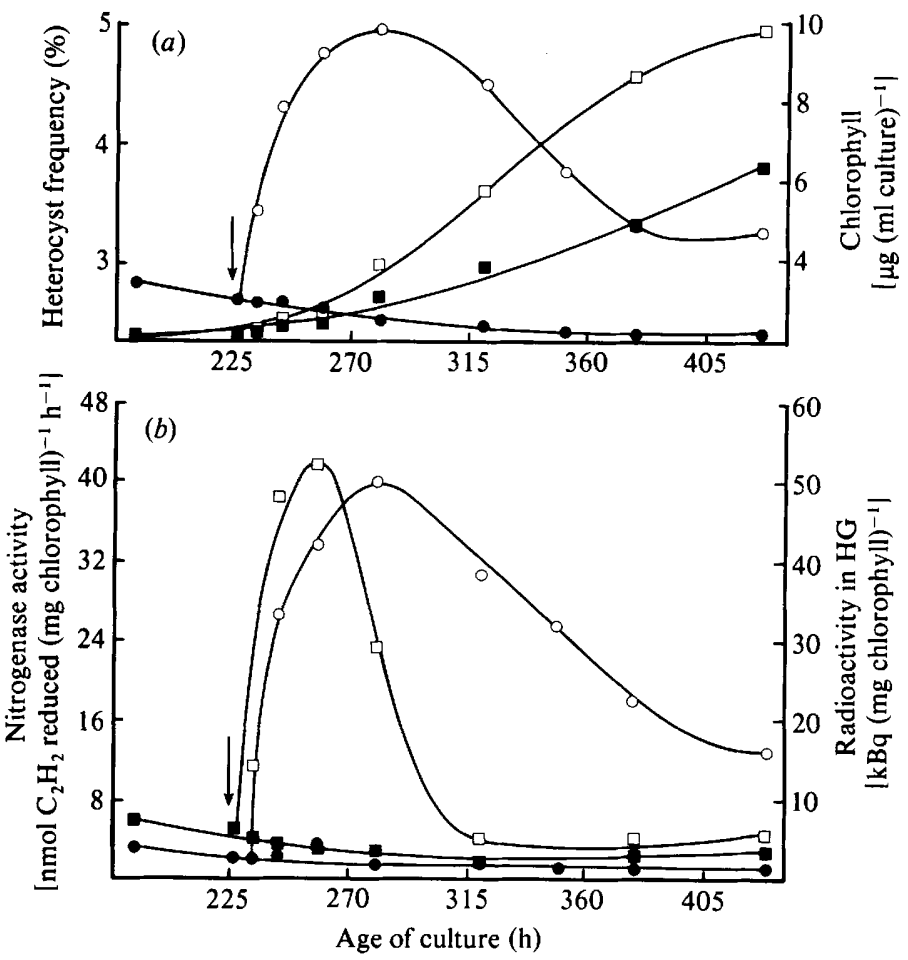

Fig. 1. Effect of induced heterocyst differentiation on A. cylindrica. (a) Total heterocyst frequency in nitrogen-free medium $(O)$ and combined nitrogen medium $(O)$, and chlorophyll level in nitrogen-free medium $(\square)$ and combined nitrogen medium ( $\square$ ). (b) Nitrogenase activity in nitrogen-free medium $(O)$ and combined nitrogen medium $(O)$, and incorporation of sodium $[1-14 \mathrm{C}]$ acetate into the heterocyst glycolipid fraction in nitrogen-free medium $(\square)$ and combined medium $(\square)$. The arrows indicate the times of transfer of cells from combined nitrogen medium to nitrogen-free medium.

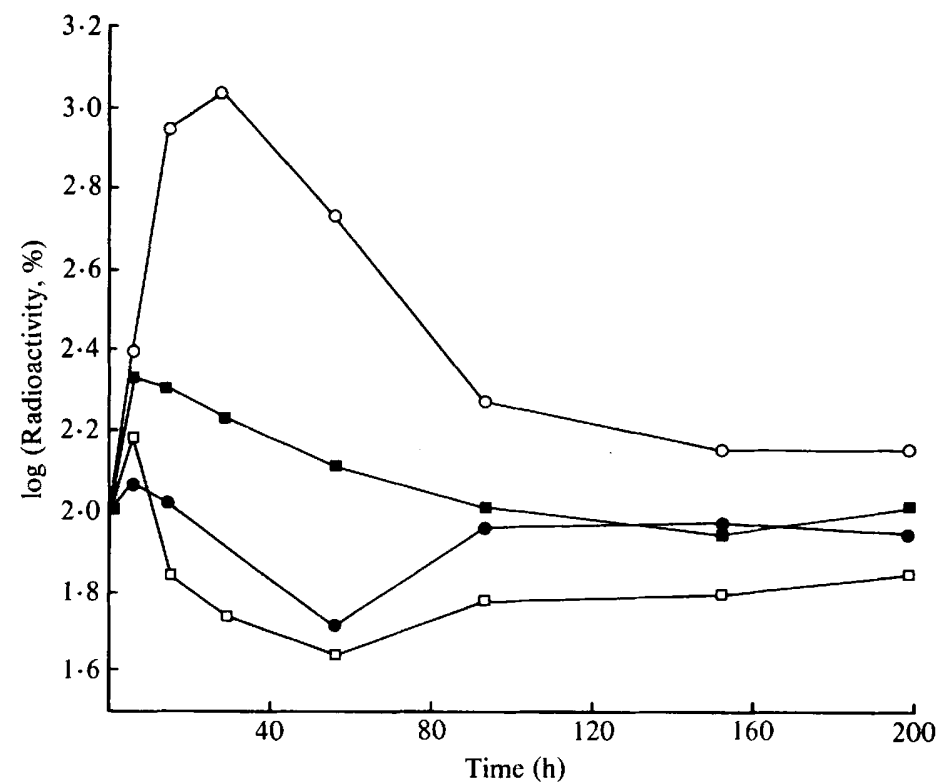

Fig. 2. Incorporation of sodium $\left[1^{-14} \mathrm{C}\right]$ acetate into the lipid fractions of $A$. cylindrica during induced heterocyst differentiation expressed as a percentage of that of a batch culture grown continuously in combined nitrogen medium. Radioactivity was measured as d.p.m. (mg chlorophyll) ${ }^{-1}$. O, HG; SQDG; $\square$, MGDG; 1 , DGDG plus PG. 

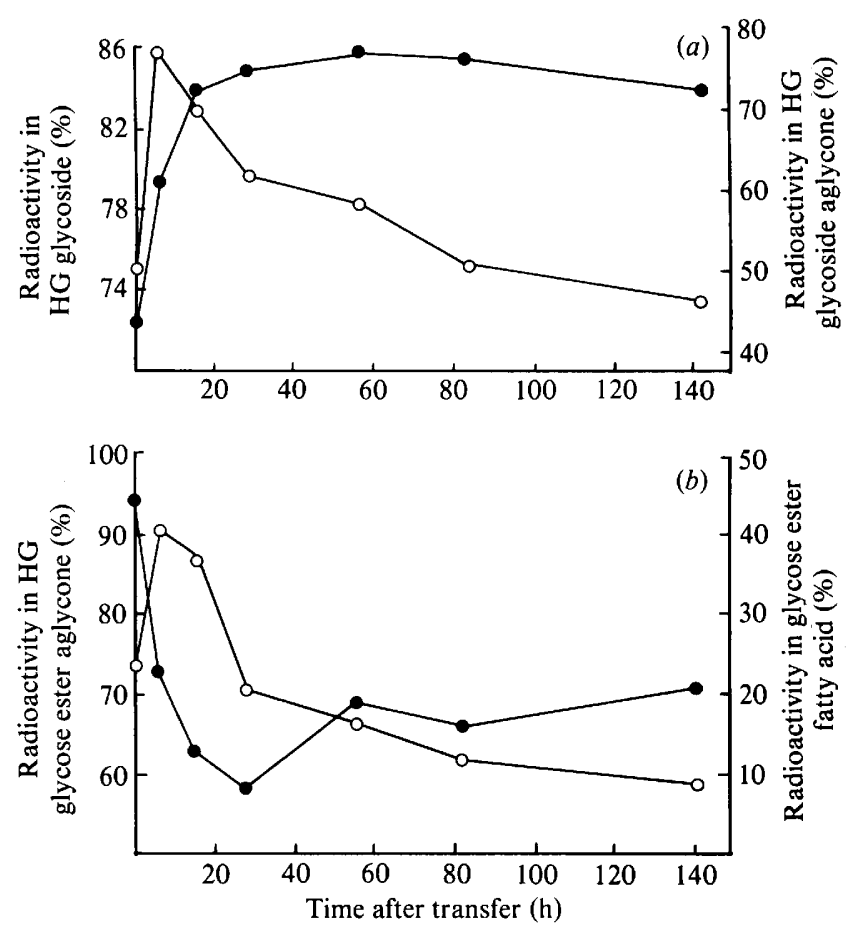

Fig. 3. Incorporation of sodium $\left[1-{ }^{14} \mathrm{C}\right]$ acetate into heterocyst glycolipid fractions during induced heterocyst differentiation. (a) $\mathrm{O}$, Radioactivity in the $\mathrm{HG}$ glycoside fraction (expressed as a percentage of total $\mathrm{HG}$ radioactivity); 0 , radioactivity in the $\mathrm{HG}$ glycoside aglycone fraction (expressed as a percentage of glycoside radioactivity). (b) $\mathrm{O}$, Radioactivity in the $\mathrm{HG}$ glycose ester aglycone fraction (expressed as a percentage of total glycose ester radioactivity);, radioactivity in the glycose ester fatty acid fraction (expressed as a percentage of glycose ester aglycone radioactivity).

that the increased labelling of the HG fraction was not due to a general stimulation of acyl lipid biosynthesis initiated by transfer, but was specifically associated with induced heterocyst differentiation.

The HG fraction has been shown in compositional anci radiolabelling experiments to be a heterogeneous group of structurally related, very long chain polyhydroxyalkaneglycosides and hydroxyfatty acid glycose esters (Lambein \& Wolk, 1973; Abreu-Grobois et al., 1977). During heterocyst differentiation induced by transfer to nitrogen free medium, incorporation of sodium $\left[1-{ }^{14} \mathrm{C}\right]$ acetate into the glycoside fraction of $\mathrm{HG}$ was maximal $6 \mathrm{~h}$ after transfer $(85.5 \%$ of total HG radioactivity, Fig. $3 a$ ), returning during the following $136 \mathrm{~h}$ to the value observed at the time of transfer. Within the glycose ester fraction the time course of radiolabelling of the fatty acyl and hydroxyfatty acyl residues, which constitute the aglycone moieties of the glycose ester fraction, followed a similar pattern to that observed for the glycoside fraction of HG (Fig. $3 b$ ). At the time of transfer, $44 \%$ of the sodium $\left[1-{ }^{14} \mathrm{C}\right]$ acetate incorporated into the aglycone residues of the glycose ester fraction was in the fatty acyl groups (Fig. $3 b$ ), the remainder being in hydroxyfatty acyl residues; the fatty acyl fraction contained no radioactive very long chain fatty acids and the distribution of label within the fatty acids $(16: 0,82 \cdot 8 \% ; 16: 1,3 \cdot 6 \% ; 18: 0,5 \cdot 2 \%$; $18: 1,8.5 \%$ ) was very similar throughout the time course of differentiation. Labelling of this fatty acyl fraction fell rapidly after transfer to nitrogen-free medium (Fig. $3 b$ ), indicating that biosynthesis of the very long chain hydroxyfatty acid moieties of the glycose ester fraction is stimulated under these conditions.

The lipid moieties of the HG glycoside fraction contained $44 \%$ of the total glycoside radioactivity in the batch culture grown in the combined nitrogen medium at the time of transfer 
Table 1. Distribution of radioactivity amongst the aglycone moieties of the glycoside fractions of the heterocyst glycolipid after transfer from combined nitrogen medium to nitrogen-free medium

\begin{tabular}{|c|c|c|c|c|c|}
\hline \multirow{2}{*}{$\begin{array}{l}\text { Time after transfer } \\
\text { to nitrogen-free } \\
\text { medium (h) }\end{array}$} & \multicolumn{5}{|c|}{ Percentage distribution } \\
\hline & Alkanetetraol & Alkanetriol & Alkanediol & Alkanol & $' X^{\prime *}$ \\
\hline 0 & $6 \cdot 6$ & $62 \cdot 7$ & $13 \cdot 7$ & $13 \cdot 4$ & $3 \cdot 6$ \\
\hline 6 & $8 \cdot 0$ & $66 \cdot 8$ & 11.9 & $11 \cdot 1$ & $2 \cdot 2$ \\
\hline 15 & $10 \cdot 6$ & $78 \cdot 4$ & $5 \cdot 2$ & $5 \cdot 3$ & 0.5 \\
\hline 28 & $11 \cdot 1$ & 78.9 & 5.0 & $4 \cdot 5$ & 0.5 \\
\hline 56 & $7 \cdot 6$ & $47 \cdot 2$ & $21 \cdot 2$ & $21 \cdot 3$ & $2 \cdot 7$ \\
\hline 93 & $5 \cdot 9$ & $47 \cdot 2$ & $22 \cdot 3$ & $21 \cdot 1$ & $3 \cdot 5$ \\
\hline 142 & $5 \cdot 7$ & $48 \cdot 9$ & $21 \cdot 2$ & $20 \cdot 9$ & $3 \cdot 3$ \\
\hline
\end{tabular}

\footnotetext{
* Component ' $X$ ' was a minor component which chromatographed between the alkanol fraction and the solvent front on silicagel-G TLC.
}

(Fig. 3a). Following transfer to nitrogen-free medium, this proportion increased rapidly to reach $72 \%$ after $15 \mathrm{~h}$ and this level was then maintained throughout the time course of heterocyst differentiation. Thus, at the maximal rates of overall HG labelling (15-28 h, Fig. 1b) synthesis of the polyhydroxyalkane components was maximal. The labelling data indicates that transfer to nitrogen-free medium causes specific activation of the enzyme systems involved in the formation of the lipid moieties of the $\mathrm{HG}$ glycoside and glycose ester fractions.

Analysis of the distribution of radioactivity amongst the polyhydroxyalkane components of the HG glycoside fraction showed that during maximal synthesis of this fraction (15-28 $\mathrm{h}$ after transfer) radioactivity in 1,3,26,28-tetrahydroxyoctacosane (alkanetetraol) and 1,3,25trihydroxyhexacosane (alkanetriol) accounted for $90 \%$ of the radioactivity in the lipid moieties (Table 1). From $56 \mathrm{~h}$ onwards, however, when overall labelling of HG was reduced (Fig. $1 b$ ), $40 \%$ of the aglycone radioactivity accumulated in fractions containing very long chain alkanols and alkanediols (Abreu-Grobois et al., 1977; Mohy-ud-Dhin, 1980) as well as their long chain $\left(\mathrm{C}_{16}\right.$ and $\mathrm{C}_{18}$ ) homologues (W. J. Krepski \& T. J. Walton, unpublished observations), and there was a concomitant reduction in the labelling of the alkanetetraol and alkanetriol fractions. Thus, in the most active phase of $\mathrm{HG}$ biosynthesis, during the early phase of heterocyst differentiation, the characteristic very long chain alkanetriols, alkanetetraols and hydroxyfatty acids are major products, while after this phase of heterocyst differentiation, when the heterocyst is functionally mature as judged by maximal rates of nitrogen fixation, synthesis of the alkanol, alkanediol and fatty acid components of $\mathrm{HG}$ becomes significant.

The biosynthesis of the very long chain lipid moieties of the heterocyst glycolipid fraction is therefore under separate control mechanisms from those regulating glycerolipid fatty acid biosynthesis. These allow a sustained increase in generation of the lipid moieties of the HG fraction during an early phase of heterocyst differentiation in $\boldsymbol{A}$. cylindrica and other cyanobacteria (Wahal et al., 1974; Feige \& Ritschel, 1978), which is characterized by formation of the $\mathrm{C}_{26}$-triol and $\mathrm{C}_{28}$-tetraol glycosides and the very long chain hydroxyfatty acid glycose esters. The regulatory mechanism also enables synthesis of $\mathrm{HG}$ to be shut down in filaments possessing physiologically mature heterocysts, and during this phase quantitatively minor very long and long chain HG components are produced. These results suggest that there is a minimum of two points at which biosynthesis of the lipid moieties of the HG glycoside fraction is regulated. In order to provide an adequate supply of very long chain precursors during deposition of the laminated layer, an enzyme system involved specifically in synthesis of very long chain acyl lipids, but separate from bulk fatty acid biosynthesis, e.g. a fatty acid elongase, must be stimulated during differentiation. In addition the observation that formation of polyhydroxyalkanes is reduced in filaments possessing mature heterocysts suggests that an aliphatic hydroxylation system is transiently activated during heterocyst differentiation. 


\section{REFERENCES}

Abreu-Grobois, F. A., Billyard, T. C. \& Walton, T. J. (1977). Biosynthesis of heterocyst glycolipids of Anabaena cylindrica. Phytochemistry 16, 351-354.

BRADLEY, S. \& CARR, N. G. (1976). Heterocyst and nitrogenase development in Anabaena cylindrica. Journal of General Microbiology 96, 175-184.

Bryce, T. A., Welti, D., Walsby, A. E. \& Nichols, B. W. (1972). Monohexoside derivatives of longchain polyhydroxy alcohols; a novel class of glycolipid specific to heterocystous algae. Phytochemistry 11, 295-302.

FAY, P. (1980). Nitrogen fixation in heterocysts. In Recent Advances in Biological Nitrogen Fixation, pp. 121-165. Edited by N. S. Subba Rao. London: Arnold.

Feige, G. B. \& Rirschel, G. A. (1978). Physiologische Aspekte der Ökologie aquatischer Blaualgen. Verhandlung der Gesellschaft für Ökologie, Kiel (1978), 303-309.

HaselkoRn, R. (1978). Heterocysts. Annual Review of Plant Physiology 29, 319-344.

HaURY, J. F. \& Wolk, C. P. (1978). Classes of Anabaena variabilis mutants with oxygen sensitive nitrogenase activity. Journal of Bacteriology 136, 688-692.

KREPSKI, W. J. \& WALTON, T. J. (1979). Effect of tryptophan analogues on lipid metabolism in the cyanobacterium Anabaena cylindrica. Biochemical Society Transactions 7, 1268-1269.

LAMbein, F. \& WoLK, C. P. (1973). Structural studies on the glycolipids from the envelope of the heterocyst of Anabaena cylindrica. Biochemistry 12, 791798.

MoHY-UD-DHIN, M. T. (1980). Studies on the biosynthesis of the heterocyst specific glycolipids of Anabaena cylindrica. Ph.D. thesis, University of Wales, U.K.

Nichols, B. W. \& Wood, B. J. B. (1968). New glycolipid specific to nitrogen-fixing blue-green algae. Nature, London 217, 767-768.

WaHAL, C. K., BHatTaCharya, N. C. \& Talpasayi, E. R. S. (1974). Lipid synthesis and heterocyst development in Anabaena ambigua Rao. Indian Journal of Experimental Biology 12, 92-93.

W ALSBY, A. E. \& Nichols, B. W. (1969). Lipid composition of heterocysts. Nature, London 221, 673674.

Winkenbach, F., Wolk, C. P. \& Jost, M. (1972). Lipids of membranes and of the cell envelope in heterocysts of a blue-green alga. Planta 107, 69-80. ZEPKE, H. D., HeINZ, E., RADUNZ, A., LINSCHEID, $M$. \& Pesch, R. (1978). Combination and positional distribution of fatty acids in lipids from blue-green algae. Archives of Microbiology 119, 157-162. 